\title{
El paradigma de paz imperfecta en un campamento de refugiados: empoderamiento pacifista de adolescentes saharauis*
}

\author{
[Versión en Castellano] \\ The Paradigm of Imperfect Peace in a Refugee Camp: Pacifist \\ Empowerment of Sahrawi Adolescents \\ O paradigma da paz imperfeita em um campo de refugiados: \\ empoderamento pacifista de adolescentes do Saarawi
}

Recibido 23 de junio, 2020. Aceptado 27 de agosto, 2020.

\author{
Diana Feo-Ardila ** \\ https://orcid.org/0000-0002-6906-2344 \\ Colombia \\ Gladys-Adriana Espinel-Rubio *** \\ https://orcid.org /0000-0002-8796-9257
}

Colombia

Para citar este artículo:

Feo-Ardila, Diana; Espinel-Rubio,

Gladys-Adriana (2021)

El paradigma de paz imperfecta en un campamento de refugiados: empoderamiento pacifista de adolescentes saharauis.

Ánfora, 28(50), 45-74.

https://doi.org/10.30854/anfv28.n50.2021.714

Universidad Autónoma de Manizales. ISSN 0121-6538.

E-ISSN 2248-6941.

CC BY-NC-SA 4.0
* Este artículo es resultado de la investigación "La construcción de una identidad colectiva en la población adolescente del campo de refugiados de Tinduf (Argelia)" del Máster en Cultura de Paz, Conflictos, Educación y Derechos Humanos, cursado en 2016-2017. Las autoras declaran que no hubo conflicto de intereses en la ejecución del proyecto de investigación.

** Artista visual. Máster en Cultura de Paz, Conflictos, Educación y Derechos Humanos de la Universidad de Granada, España; maestranda en Problemáticas Sociales Infanto Juveniles de la Universidad de Buenos Aires, Argentina. Miembro del Grupo de Investigación en Comunicación y Medios (GICOM) de la Universidad Francisco de Paula Santander (UFPS), Colombia. Asesora y consultora en procesos comunitarios con población víctima del conflicto armado interno y/o en riesgo de estarlo y con comunidades urbanas, rurales e indígenas en condiciones de vulnerabilidad social. Con trayectoria investigativa en temas de identidad y migración con niños, niñas y adolescentes. Correo electrónico: dianafeoa@gmail.com

\footnotetext{
*** Comunicadora social-periodista. Magíster en Estudios sobre problemas políticos latinoamericanos de la Universidad del Cauca. Doctoranda en Ciencias Políticas de la Universidad del Zulia, Venezuela. Docente asistente del Departamento de Pedagogía, Andragogía, Comunicación y Multimedia de la Universidad Francisco de Paula Santander (UFPS), Colombia. Directora del Grupo de Investigación en Comunicación y Medios (GICOM). Correo electrónico: gladysespinel@ufps.edu.co
} 


\title{
Resumen
}

Objetivo: identificar el paradigma de la paz imperfecta sustentado en el empoderamiento pacifista, construido a través de la identidad de resistencia y la transmisión de la memoria histórica en un grupo de adolescentes residentes en el campamento de refugiados saharauis en Tinduf, Argelia. Metodología: se trata de una investigación cualitativa, enfocada desde el construccionismo social. Se utilizaron como instrumentos de recolección de información la fotoelicitación, la cartografía social y la creación artística, además de grupos focales y entrevistas semiestructuradas. Resultados: se recuperaron las narrativas, sentires, inquietudes y vivencias de nueve adolescentes, reconociéndolos como sujetos portadores y generadores de conocimientos, que dan cuenta de sus percepciones y alternativas sobre su pasado, presente y futuro. También se halló que la historia de resistencia del pueblo saharaui se constituye en ejemplo de empoderamiento pacifista, pues éstos se reconocen y recrean cotidianamente desde sus prácticas discursivas y sus apuestas comunitarias pacíficas, así como en el fortalecimiento de su tejido social, basado en valores como la cooperación y la solidaridad. Conclusiones: se concluye que la paz imperfecta se dilucida como un proceso y/o un camino inacabado, en dónde las realidades sociales y ambientales evolucionan, estando en continuo movimiento, así como las conflictividades.

Palabras-clave: Adolescentes; Refugiados; Paz imperfecta; Empoderamiento; Memoria histórica; Identidad; Solidaridad internacional; Investigación sobre la paz.

\begin{abstract}
Objective: to identify the paradigm of imperfect peace based on pacifist empowerment, built through the identity of resistance and the transmission of historical memory in a group of adolescents residing in the Sahrawi refugee camp in Tindouf, Algeria. Methodology: this qualitative research focused on social constructionism. Photo-elicitation, social cartography and artistic creation were the data collection instruments, as well as focus groups and semi-structured interviews. Results: the narratives, feelings, concerns and experiences of nine adolescents were collected, recognizing them as carriers and generators of knowledge, who account for their perceptions and alternatives about their past, present and future. It was also found that the history of resistance of the Saharawi people constitutes an example of pacifist empowerment, since they are recognized and recreated daily from their discursive practices and their peaceful community commitments, as well as in the strengthening of their social fabric, based on values such as cooperation and solidarity. Conclusions: it is concluded that imperfect peace is elucidated as a process
\end{abstract}


and or an unfinished path, where social and environmental realities evolve, being in continuous movement, as well as the conflicts.

Keywords: Adolescents; Refugees; Imperfect peace; Empowerment; Historical memory; Identity; International Solidarity; Research on peace.

\section{Resumo}

Objetivo: identificar o paradigma da paz imperfeita sustentado pelo empoderamento pacifista, construído através da identidade da resistência e da transmissão da memória histórica em um grupo de adolescentes residentes no campo de refugiados do Saarawi em Tinduf, Argélia. Metodologia: trata-se de uma pesquisa qualitativa, com enfoque no construcionismo social. A foto-elicitação, a cartografia social e a criação artística foram utilizadas como instrumentos de coleta de informações, bem como grupos focais e entrevistas semiestruturadas. Resultados: as narrativas, sentimentos, preocupações e experiências de nove adolescentes foram recuperadas, reconhecendo-os como portadores e geradores de conhecimento, que respondem por suas percepções e alternativas sobre seu passado, presente e futuro. Verificou-se também que a história de resistência do povo saharaui constitui um exemplo de empoderamento pacifista, visto que são reconhecidos e recriados diariamente a partir das suas práticas discursivas e dos seus compromissos pacíficos comunitários, bem como no fortalecimento do seu tecido social, com base em valores como cooperação e solidariedade. Conclusões: conclui-se que a paz imperfeita é diluída como um processo e/ou um caminho inacabado, onde as realidades sociais e ambientais evoluem, estando em movimento contínuo, bem como conflitos.

Palavras-chave: Adolescentes; Refugiados; Paz imperfeita; Empoderamento; Memória histórica; Identidade; Solidariedade Internacional; Pesquisa de paz. 


\section{Introducción}

La paz imperfecta planteada por Muñoz (2001) facilita la comprensión de las tensiones que emergen entre las vías pacíficas en relación con las violentas y las mediaciones sociales que se dan en tales circunstancias. Así, reconoce que todos los grupos humanos tienen capacidades para el desarrollo de la paz, haciendo uso del empoderamiento pacifista; es decir, el ejercicio del poder integrativo (Boulding, 1993) o desde las paces como decisión interna y personal para actuar y transformar su entorno, al tomar conciencia de las capacidades para la paz y avalando las potencialidades de otros seres para su desarrollo (Loaiza, 2016).

Bajo esta perspectiva, el presente artículo identifica el paradigma de la paz imperfecta sustentado en el empoderamiento pacifista, construido a través de la identidad de resistencia y de la memoria histórica en un grupo de adolescentes residentes en el campamento de refugiados saharauis en Tinduf, quienes en sus relatos expresaron concepciones frente a su situación de refugio y exilio, así como la historia de migración forzada y el conflicto de su pueblo, lo cual ha transformado imaginarios y entornos, generando tensiones y fragmentación del tejido social que son subsanados por la decisión de convivir en paz.

La Declaración de Cartagena (Refworld, 1984) define a los refugiados como personas que han huido de su país porque su vida, seguridad o libertad han sido amenazadas por la violencia generalizada, la agresión extranjera, los conflictos internos, la violación masiva de los derechos humanos u otras circunstancias que hayan perturbado gravemente el orden público.

Por la condición de los campamentos que, en su mayoría, son zonas cerradas y delimitadas donde se ha configurado una especie de relación de asistencia y protección entre los refugiados y quienes los asisten, se han venido construyendo discursos alrededor del refugiado, ya sea como víctimas o como receptores de ayuda humanitaria; esto ha generado la estigmatización como carentes y limitados, subestimando o desconociendo (en algunos casos) la capacidad de gestión y empoderamiento de sus habitantes (Valdivia, 2013).

No obstante, en el campamento de refugiados saharauis -un pueblo que a pesar de vivir el drama del exilio y enfrentarse diariamente a la vulneración sistemática de sus derechos humanos- se ha demostrado interés por encontrar una solución pacífica al conflicto, convirtiendo esta resistencia en una iniciativa de paz imperfecta. Esto demuestra "que los fenómenos de la paz y la violencia co-existen (probablemente en distintas escalas y manifestaciones); e incluso, en las situaciones más adversas e infames es posible hacer emerger la vida y desplegar la paz” (Loaiza, 2016, p. 63). 
Ante este escenario, es conveniente hablar de la paz entendida como construcción cultural y elemento constitutivo de las realidades sociales y como un símbolo de interpretación y acción, donde se ven involucrados plexos de emociones y de cogniciones subjetivas e intersubjetivas (Muñoz y Martínez, 2011). Se plantea, entonces, la importancia de reconocer "aquellos espacios e instancias en las que se pueden detectar acciones que crean paz, a pesar de que estén en contextos en los que existen los conflictos y la violencia” (López, 2011, p. 9), incluyendo y reconociendo desde una perspectiva de conflicto las diferentes experiencias de paz, tanto de los individuos como de los grupos humanos.

En el marco de esta investigación se identificaron las experiencias de empoderamiento pacifista en un grupo de adolescentes, reconociéndolos como portadores de información y su importancia en la construcción de conocimiento, saberes y prácticas discursivas. Además, entendiendo la adolescencia como una imagen colectiva que se modifica históricamente dependiendo de la situación estructural en que se encuentra, que da cuenta de diversas experiencias y representaciones sobre lo que significa ser adolescente en distintos contextos socioculturales, así como desde las dimensiones de diversidad y desigualdad social (Colangelo, 2014).

Los estudios sobre el fenómeno migratorio trasnacional y la situación de refugio atravesados por la perspectiva y el paradigma de la paz imperfecta, son relativamente recientes y sus avances desde la investigación empírica, la comprensión teórica y la orientación metodológica están en desarrollo; estos escenarios necesitan ser abordados de manera integral teniendo en cuenta diversas dimensiones para su comprensión (Sandoval, 2009; Egea y Soledad, 2011).

Por lo anterior, este artículo buscó responder a las preguntas ¿De qué manera se expresa el paradigma de la paz imperfecta en los adolescentes saharauis en el campamento del Tinduf?, ¿Hay una configuración de empoderamiento pacifista?, ¿Mediante qué mecanismos la memoria histórica y las identidades de resistencia recrean las condiciones para comprender la paz como un proceso y/o un camino inacabado, entendiendo que las realidades sociales y ambientales evolucionan y están en continuo movimiento, así como las formas conflictivas?

\section{Antecedentes y marco teórico}

\section{Investigaciones sobre los saharauis en los campamentos.}

Hasta la primera década del siglo XXI, la investigación académica en torno a los campamentos de refugiados de Tinduf era escasa. Canales (2009), señaló que los campamentos son una "parte dramática del pueblo saharaui con poca referencia bibliográfica” (p. 1). Los Saharauis (The western Saharans), escrito por 
Hodges (1984) y traducido por Portillo (2014) es hasta ahora el documento más significativo publicado en Español. El informe de Hogdes fue encargado y publicado, en su momento, por The Minority Rights Group (MRG) para promover en la opinión pública la comprensión de problemática.

La investigación de Hodges tiene vigencia comprobada, pues en el 2012, el Colegio de Abogados de Nueva York la utilizó en su Informe sobre los aspectos jurídicos del conflicto del Sáhara Occidental (Portillo, 2014).

Otros referentes sobre los saharauis son informes de ONGs, agencias de cooperación y documentos oficiales de la ACNUR y la OIM. Además, de reportes de los medios masivos de comunicación, pero en la última década (2010 hasta el presente) se han registrado importantes avances, sobre todo en comprensión del proceso enseñanza y aprendizaje al interior del campamento.

Pozuelo y Gimeno (2010), destacan por la teoría comprometida en el análisis postcolonial y la práctica antropológica a través de las investigaciones realizadas por equipos mixtos: Saharauis y no saharauis y mencionando los trabajos de Mato (2000) y Gimeneo y Ali (2007), además Pozuelo y Gimeno (2010) manifiestan que se han realizado estudios sobre la juventud saharaui en $2005 \mathrm{y}$ la posible creación de una universidad del desierto en 2009, además de pesquisas sobre la realidad del Sáhara (en 2008), el proyecto Vacaciones en Paz para los niños saharauis (en 2006), investigaciones del mercado laboral (2007), estudios interesados en la poesía oral saharaui (2009) y la recuperación científica de los licenciados saharauis en Cuba en 2010 (Pozuelo y Gimeno, 2010, p. 2).

Por su parte, Aranda, Arias y González (2012) describieron el proyecto de formación del profesorado saharaui en los campamentos; Jiménez (2015), en su tesis doctoral estudió el perfil, contexto, competencias, necesidades e intereses de los docentes que enseñan el español; Calero, Cruz y Campos (2016) y Abdala (2017) presentaron sendos estudios sobre la formación de promotoras de salud y sobre prácticas educativas inclusivas en la República Árabe Saharaui Democrática (RASD).

\section{Apuestas de empoderamiento pacifista.}

Tanto empoderamiento pacifista como el paradigma de la paz imperfecta son categorías conceptuales de reciente aparición y estudio. Las investigaciones más destacadas en Colombia son las adelantadas por Anacona (2019), Fernández (2018), Hernández, (2014, 2015).

Anacona (2019) propuso el diálogo entre la perspectiva de la paz imperfecta y paradigma decolonial para explicar el proceso de empoderamiento pacifista de las mujeres del cabildo indígena Yanakuna de Cali, Colombia. En su trabajo, la investigadora trianguló epistemológica y metodológicamente la cosmovisión 
de la Chakana o Cruz del Sur, con la investigación-acción-participación y el feminismo decolonial comunitario. Gracias a esto encontró concepciones de paz como principio que moviliza e integra de su pueblo y de acciones de noviolencia, expresadas, por ejemplo, a través del tejido, "las mujeres señalan que la construcción de paz es una reflexión constante que implica deshilar cuando sea necesario para siempre buscar el mejor tejido" (Anacona, 2019, p. 452).

Fernández (2018) estudió el empoderamiento pacifista de las mujeres afrodescendientes de La Toma, en Suárez, Cauca (Colombia). Concluyó que ellas optaron por usar mecanismos noviolentos como movilizaciones, escenarios solidarios para la planeación del territorio y mecanismos jurídicos para la protección de sus derechos, pese a vivir en un contexto con expresiones de violencia. Así, logran resinificar el poder con la puesta en práctica de "sus potencialidades y capacidades para el diálogo, la concertación y movilización de procesos con los que comparten cosmovisiones propias de las comunidades negras" (p. 97), es decir, propias de su identidad colectiva.

Por su parte, Hernández (2015) explicó el proceso de paz entre el gobierno colombiano y las Farc-Ep como una apuesta de empoderamiento pacifista. Destacó la transformación generada por este empoderamiento: el encuentro de orillas ideológicas y militares divergentes, el diálogo entre enemigos, la definición de una hoja de ruta de la negociación, la superación de los estancamientos y saboteadores, y el alcance de acuerdos en lo general y parcial, que permiten la configuración de una nueva realidad. El empoderamiento pacifista es definido acá, primero, como la toma de conciencia de las capacidades que se tienen para la transformación pacífica de los conflictos y, segundo, como "los procesos en que la paz, la transformación pacífica de los conflictos, la satisfacción de necesidades o el desarrollo de capacidades ocupan el mayor espacio personal, público y político posible" (Hernández, 2015, p. 183).

En su tesis doctoral "Empoderamiento pacifista de experiencias comunitarias locales en Colombia (1971-2013)”, Hernández (2014) abordó las experiencias de resistencia civil como expresión del empoderamiento pacifista porque hay generación y apropiación de poder noviolento. Entonces, quienes resisten, en este caso: el Consejo Regional Indígena del Cauca (CRIC) y la Asociación de Trabajadores Campesinos del Carare (ATCC), reconocen su poder y potencialidad para el cambio y la acción, sumado a su posibilidad de oponerlo al poder dominante. Además, definió el empoderamiento pacifista como:

Procesos perfectibles que permiten el desarrollo de capacidades y competencias, individuales, comunitarias o colectivas para construir paces imperfectas o inacabadas; reconocen y posicionan experiencias de la misma naturaleza; apropian y fomentan un concepto de poder noviolento en sus formas organizativas propias, 
su participación social y política, al diseñar un futuro más democrático y pacífico; y crean espacios de interacción y diálogo para la transformación pacífica de conflictos. Por todo ello, otorgan poder a la paz (Hernández, 2014, p. 22).

Sobre el empoderamiento pacifista como concepto y sus desarrollos en América Latina y México, Sandoval (2015) concluyó que éste se basa en pensar, sentir, actuar de manera diferente, de modo que se transformen socialmente las condiciones de manera noviolenta "teniendo como base el conocimiento, el respeto y la aplicación de los derechos humanos, la búsqueda de la justicia social y la libertad en la sociedad de la que forme parte" (p. 93).

En cuanto, al involucramiento de los niños, niñas, adolescentes y jóvenes en los procesos de construcción de paz, las investigaciones son diversas y dispersas, pues son enunciados e interpretados desde un amplio espectro categorial; se abordan desde la acción colectiva, la agencia política, las subjetividades y las territorialidades componen el repertorio conceptual en el que se enmarcan estas investigaciones. Lo cierto, es que buena parte, destacan el carácter situado de las experiencias que analizan, siendo un factor determinante para su comprensión, como es el caso de este artículo. En Colombia se destacan los trabajos de Posada et al. (2018); Salas, Valenzuela y Prada (2019); Parra (2014) y Alvarado, Patiño y Loaiza (2012).

En relación con las experiencias de construcción de paz en una situación de refugio, se destacan los trabajos de Romariz (2012), "Tú tienes tu Líbano y yo tengo el mío: desplazamiento inducido por el conflicto e identidad” allí explicó lo fundamental que ha sido la transmisión de la memoria histórica a los adultos libaneses que se han tenido que criar en el extranjero, luego de que sus padres se vieran abocados a una migración y exilio forzoso durante la guerra que tuvo lugar en el Líbano entre 1975 y 1990. Y Valdivia (2013), quien analizó la experiencia del grupo musical Sierra Leone's Refugee All Stars como refugiados en los campamentos de Kalia y Sembakounya, en Guinea después de la guerra civil de Sierra Leona de 1991 a 2001.

\section{La paz imperfecta, memoria histórica e identidad de resistencia}

Fisas (1998), aclaró que la paz es algo más que la ausencia de conflicto armado; tiene que ver con superar, reducir o evitar de todo tipo de violencia y con la capacidad que pueden tener los ciudadanos de transformar estos conflictos en oportunidades creativas de encuentro, comunicación, cambio, adaptación e intercambio, lo que permitirá la construcción de una cultura de paz. Sin embargo, este proceso no es sencillo. Para Mayor (1994), ir de la guerra a la paz comprende la transformación de una sociedad dominada por el Estado, como único garante de 
la seguridad en un mundo con riesgos, a una sociedad civil, "en la cual las personas trabajan, crean y desarrollan la urdimbre de su existencia en comunidades liberadas de los temores inherentes a una cultura bélica” (p. 16).

El planteamiento de entender la paz desde una perspectiva compleja y conflictiva, reconociendo las formas en que los seres humanos, frente a situaciones de violencia extremas, actúan de manera cooperativa y solidaria, abre el diálogo científico en el marco de la investigación para la paz a un nuevo paradigma, al que Muñoz (2001) denominó paz imperfecta o inacabada.

Muñoz (2001) plantea una ruptura en las concepciones de una paz perfecta y utópica, y en contraparte reconoce las prácticas pacíficas donde ocurren, ayudando a planificar futuros conflictivos. Esta perspectiva se ha constituido en un instrumento la investigación para la paz, pues incorpora el debate y construcción de nuevos paradigmas para comprender y construir mundos más pacíficos, justos y perdurables en el tiempo, con mayor equidad entre los géneros y las etnias (Muñoz, 2001).

Así, la paz imperfecta se podría entender como:

...todas estas experiencias y estancias en la que los conflictos se han regulado pacíficamente, es decir en las que los individuos y/o grupos humanos han optado por facilitar la satisfacción de las necesidades de los otros, sin que ninguna causa ajena a sus voluntades lo haya impedido (Muñoz, 2001, p. 14).

Puesto que la paz imperfecta está cotidianamente presente en la vida y todos los seres humanos tienen un potencial enorme para la construcción de paz, un nuevo reto del investigador social es identificar espacios y situaciones en diferentes contextos donde los seres humanos construyan, fomenten y potencien relaciones positivas con los demás en pro de una convivencia justa y pacífica.

Muñoz (2001) propone la generación de una conexión entre pasado, presente y futuro, donde los procesos de construcción, recreación y alimentación de identidades colectivas, en este caso de los refugiados, se pueden constituir como factores claves para el reconocimiento de su ejercicio ciudadano, de sus derechos humanos y de la recuperación de su dignidad humana, así como en una forma de empoderamiento pacifista y mediaciones entre conflictividades y prácticas pacifistas.

Muñoz y Martínez (2011) entienden el poder como un aspecto intrínseco de las relaciones humanas, por tanto, todas las personas y los grupos tienen poder y lo ejercen de diversas formas e intensidad.

En este punto, se puede decir que todas las condiciones humanas (capacidades, potencialidades, proyectos, necesidades y conflictos) y formaciones sociales (culturas, religiones, migraciones, paz, violencia) Muñoz y Martínez (2011), así 
como las circunstancias, vivencias y contexto hacen parte de nuestra construcción identitaria, y el uso del poder o empoderamiento, deben aportar al reconocimiento y mantenimiento de dicha identidad ya sea individual o colectiva, fortaleciendo la capacidad para comprender y transformar las dinámicas y cambios sociales (según sea necesario y posible). Empoderarse, tal como expresa Muñoz (2001), supone una negociación con las diversas organizaciones e instituciones del entorno.

De allí la importancia de apropiarse de una concepción pacifista del poder y de la capacidad de ejercerlo ya sea individual o en grupo, donde la lucha por la garantía de sus derechos tiene una estrategia noviolenta como una decisión ética y racional pues se renuncia a la violencia, "porque están convencidos de ello o porque en su análisis de las ventajas y desventajas llegan a la conclusión de que la noviolencia acarreará más beneficios para evitar una escalada negativa del conflicto” (Girela, 2016, p.125). Así, los esfuerzos y procesos están encaminados hacia la construcción de una realidad más pacífica, reconociendo todas aquellas capacidades, prácticas y acciones que aporten al fortalecimiento de un entorno en paz y un tejido social, en el cual los actores se interesan en la promoción y mantenimiento de este entorno.

En el caso de los saharauis estos procesos de resistencia y empoderamiento pacifista se han venido desarrollando en los últimos años tras la firma de los acuerdos de paz en 1991, fortalecidos en un espacio físico como es el campamento. Según Castillejo (2000), en el campamento se transforma la concepción de transitoriedad y se configura en un entorno. Es allí, donde se comienza, pese a la falta de certeza en el futuro. El campamento se constituye en un lugar de vivencias y experiencias de empoderamiento donde "las personas negocian su existencia, sus espacios de significado y su identidad" (Valdivia, 2013, p. 136). Así, los campamentos de refugiados se asumen como espacios liminales donde se configuran significaciones y relaciones. Estos últimos, germinan en la memoria, entendida como un proceso que concede sentido y significado, un "proceso intersubjetivo, de diálogo entre individuos y sociedad, todo esto enmarcado en un sistema de valores y creencias y materializado en experiencias y representaciones (Carrizosa, 2011, p. 38).

Así, el pasado no invade el presente, sino que lo informa, fortaleciendo el sentido de pertenencia del individuo y las comunidades. Por ello, si se reconoce el carácter social y colectivo, la memoria se constituye como un elemento de la identidad (tanto individual como colectiva), ya que es determinante en la forma en que se aprehende y se construye la subjetividad. Por todo esto,

La memoria, aparte de las construcciones que cada individuo produce y comparte, está arraigada y situada allí donde se comparten espacios, lazos de pertenencia, 
solidaridades y sociabilidades. La comunidad es el escenario donde tienen lugar y se sustentan los juegos de lenguaje que hacen parte de una forma de vida, es allí donde se construyen las expresiones y acciones con que los sujetos enfrentan la adversidad y en este caso construyen memoria (Carrizosa, 2011, p. 38).

La identidad en el marco de este estudio, se abordó desde un enfoque sociológico y antropológico, estableciendo una relación directa con el discurso de los sujetos (su experiencia personal) y la interacción social, Giménez (2000). El nosotros que de allí resulta sirve para avanzar en la constitución de una acción común, necesaria para conformar el patrimonio social. Berger y Luckmann (1999) afirman que es a través de los procesos de socialización que una persona constituye su identidad, y esto es en función del grupo social en el que históricamente dicho proceso se realiza, es decir, se configura una relación dialéctica entre el individuo y su contexto social.

Planteada así la cuestión, es pertinente introducir el concepto de identidades colectivas (Giménez, 2009) centrado en el desarrollo de la concepción de identidad de resistencia en el marco de los estudios para la paz; se reconoce la importancia de la configuración de las identidades de los refugiados rescatando la diversidad existente y, sobre todo, valorando las diferencias y los aportes frente a la necesidad de lograr una convivencia pacífica entre los seres humanos.

Castells (1999), plantea que la identidad de resistencia es concebida por aquellos actores que se encuentran en algunas condiciones de subvaloración, estigmatización, exclusión social y/o dominación (política), descripción que se acerca considerablemente a la realidad del campamento de refugiados saharauis. En esos contextos se apela a la identidad como defensa de la comunidad y construyen espacios de resistencia, oposición, alternativa y supervivencia como formas de autoidentificación, a partir de diversos elementos culturales o materiales de su historia territorial, nacional, étnica, entre otras. De esta manera, los grupos sociales o comunidades marginadas, continuando con el planteamiento de Castells (1999), resisten con lo que tienen porque no pueden hacerlo como ciudadanos, porque como ciudadanos son minorías que no tienen sus derechos representados.

\section{El contexto histórico y la realidad de los protagonistas de esta investigación}

La historia del pueblo saharaui está dispersa, por lo cual, la presente aproximación sobre el origen del conflicto y su devenir, se hizo con base en fuentes primarias (entrevistas realizadas a actores sociales y políticos en el campamento) y la página web de los saharauis, relacionando esta información con la ofrecida 
por la Misión de las Naciones Unidas para el Referéndum del Sáhara Occidental (MINURSO).

Después de haber estado bajo el control colonial de España hasta 1958, durante la década de los 60, pretendieron acceder a su derecho a la libre determinación y conseguir su independencia por medio de la realización de un referéndum como la mayoría de los pueblos africanos que se encontraban bajo el dominio de alguna potencia europea. Sin embargo, ante las dificultades para culminar este proceso con éxito, deciden organizarse militarmente, dando nacimiento, en 1975, al Movimiento para la Liberación del Sahara Occidental, denominado Frente Polisario, siendo protagonistas de la lucha por su independencia en el plano popular, militar, político y diplomático.

España no otorgó la soberanía y permitió la intervención de Marruecos y Mauritania quienes asumieron una nueva potestad. Pese a ello, el 27 de febrero de 1976, mismo día que el último soldado español abandona el territorio, nace La República Árabe Saharaui Democrática (RASD), proclamada oficialmente en Bir Lehlu.

En 1979, Mauritania firmó la paz con el Frente Polisario, retirándose y renunciando a cualquier intensión sobre el territorio, coyuntura que aprovecha el gobierno marroquí, gracias al apoyo militar, político y económico de algunas potencias, mantiene la ocupación ilegal del territorio y la guerra contra el Frente Polisario, propiciando la migración y el exilio de gran parte de la población saharaui. Entre 1980 y 1984, Marruecos construye un conjunto de siete muros defensivos (con una longitud superior a los $2.720 \mathrm{~km}$.) con el objetivo de crear una línea divisoria entre los territorios invadidos por ellos y los territorios liberados por el Frente Polisario. Estos muros custodiados por aproximadamente 100 mil soldados y donde sembraron más de 7 millones de minas antipersona alrededor de ellos, se consolidó en una zona militar que dificultó el regreso de los refugiados saharauis a su legítimo territorio, presentándose diferentes violaciones a los derechos humanos en el marco de una guerra que se extendió hasta finales de 1989.

En septiembre de 1991, firmaron los acuerdos de paz mediante resolución 690 del Consejo de seguridad de la ONU, iniciando un alto al fuego con el fin de resolver la soberanía del RASD, impulsando la celebración de un referéndum de autodeterminación. Es trascendente destacar que, desde la firma de los acuerdos de paz, el pueblo saharaui decidió no mantener presos políticos y ni realizar ningún ataque armado ni violaciones al derecho internacional humanitario, fortaleciendo la idea de resistencia civil en búsqueda de una resolución final al conflicto por vías pacíficas y noviolentas.

En la actualidad, más de 200 mil saharauis, continúan exiliados residiendo en alguna parte del desierto del Sahara Argelino. En el campamento más anti- 
guo del mundo, sus habitantes evocan simbólicamente el recuerdo de su país de origen, por medio de la distribución administrativa del espacio en cinco wilayas (provincias), que llevan los mismos nombres de las ciudades de la República Árabe Saharaui Democrática. En cada Wilaya se vive a diario la precariedad, el agua, la luz eléctrica y los alimentos son cada vez más escasos, situación que empeora por las difíciles condiciones climáticas del territorio donde están asentados

\section{Metodología}

Esta es una investigación de tipo cualitativo basada en la realidad de 9 adolescentes entre los 11 y los 16 años quienes voluntariamente aceptaron participar en las actividades ${ }^{1}$, residentes en el campamento de refugiados Tinduf, seleccionados a través de un muestreo por conveniencia no probabilística por Hartani Buseif, director del centro de enseñanza media Jalil Sidi Mohamed ubicado en la Wilaya de Bojador. Ellos son: Tislem (14 años), Embarka (13 años), Bichu (13 años), Mariam (16 años), Nayat (15 años), Mohamed (14 años), Nafi (13 años), Chej (12 años) y Elhga (15 años), quienes compartían el perfil necesario para el presente estudio: habían nacido y vivido toda su vida en el campamento, se reconocen como saharauis, estaban escolarizados en el momento de la investigación y comprendían y hablaban castellano en forma fluida, se constituyen en el esqueleto y sobre todo corazón de este estudio.

El enfoque metodológico de la investigación es el construccionismo social, con base en Berger y Luckmann (1995), para comprender cómo los adolescentes se autoproducen socialmente, al mismo tiempo que crean y resignifican los marcos simbólicos de la cultura y el mundo de las relaciones sociales desde el lenguaje, permitiendo acercarse de manera fidedigna y propias narrativas, a sus concepciones con relación a su contexto y la forma en que ellos/as han construido su realidad desde la situación de refugio, intentando comprender su experiencia vivida y la realidad social a través de la interpretación de lo expresado (verbal, escrito y gráfico). De esta manera se logró comprender la importancia de la transmisión de la memoria histórica, no sólo con respecto a una realidad presente, sino frente a sus creencias, formación política e identidades, así como reconocer a los adolescentes como interlocutores válidos y productores constantes de conocimiento.

1. En relación con el consentimiento informado, el aval se obtuvo con el director de la institución educativa quien seleccionó y consintió la participación de los y las adolescentes, enmarcando la propuesta de investigación como una actividad de la institución educativa. Sumado a esto, se contó con el aval, acompañamiento y evaluación de la Asociación de Amistad con el Pueblo Saharaui de Sevilla, España (AAPSS). 
El trabajo de campo se desarrolló en cuatro fases:

1. Contacto con líderes, profesores y adultos significativos por medio de conversaciones informales para la creación de relaciones de confianza con la comunidad, así como con el grupo de adolescentes con quienes se realizaría la recolección de información. Tras explicarles a los participantes la propuesta de intervención artística, enmarcado en la técnica de grupos focales, se realizaron la cartografía social, los talleres de escritura creativa y la fotoelicitación. Con ello se logró observar y precisar las percepciones que tienen los participantes sobre su contexto en el campamento y lo que ellos conocen sobre la historia del conflicto y la migración forzada del pueblo saharaui.

2. Desarrollo de talleres de creación audiovisual colectivos para la realización de filminutos facilitó la comprensión relacional entre la memoria histórica transmitida por los adultos a los adolescentes y la configuración de sus identidades colectivas enmarcadas en su pasado, presente y futuro.

3. Aplicación de entrevistas individuales a profundidad semiestructuradas, las cuales, basándose en el análisis de narrativas, brindaron información detallada sobre sus saberes y percepciones de su entorno e historia (propia y de su pueblo) que permitieron detectar elementos en común relacionados con la configuración de sus identidades colectivas, así como en la construcción de su discurso referente a lucha de su pueblo y procesos de empoderamiento pacifista. Como cierre del trabajo de campo, se proyectaron las fotografías y filminutos, con el fin que los participantes conocieran los productos audiovisuales creados por ellos mismos, y reconocieran su capacidad creativa como portadores y generadores de conocimiento individual y colectivo.

4. De manera transversal y continua, gracias al apoyo y acompañamiento de la Asociación de Amistad con el Pueblo Saharaui de Sevilla (AAPSS), se realizaron visitas y entrevistas a integrantes de diferentes organizaciones e instituciones públicas como la delegación del Frente Polisario en Andalucía, Ministerio de Juventud y Deportes, Consejo de Derechos Humanos, Asociación de Familiares de Presos y Desaparecidos Saharauis (AFAPREDESA), Ministerio de Educación, la organización de mujeres saharauis, Nova Sahara Occidental, Ministerio de Justicia y el hospital 
de Bojador, lo cual permitió acceder a información complementaria sobre las dinámicas sociales y políticas del pueblo saharaui.

Es importante anotar que el material audiovisual producido por los y las adolescentes se presentó a los adultos significativos de la comunidad, permitiendo que ellos y ellas reconocieran su capacidad creativa como portadores y generadores de conocimiento individual y colectivo.

Para analizar la información, se utilizó el proceso de inducción propuesto por Bartolomé (1990). Las categorías definidas son: la condición de refugiado, identidad de resistencia, transmisión de la memoria histórica y procesos de empoderamiento pacifista.

Como técnica de análisis se utilizó al análisis crítico del discurso donde se define el discurso como una práctica social (Santander, 2011) con un enfoque sociocongnitivo, resaltando que los discursos son prácticas que generan realidades sociales, en el presente análisis se logró develar las formas de dominación que los adolescentes conciben frente al pasado de su pueblo, así como las formas de resistencia desarrolladas en los y las adolescentes basados en la configuración de sus identidades colectivas y en la transmisión de la memoria histórica y las relaciones de poder entre los discursos y los significados atribuidos. En este punto, emergieron prácticas discursivas que se pusieron en circulación con base en las categorías propuestas.

\section{Resultados}

\section{Experiencias de (des) arraigo de los adolescentes saharauis: entre el refugio y el retorno}

Los conocimientos que tienen los adolescentes sobre el conflicto y la migración forzada de su pueblo, están basados en los actores que iniciaron el conflicto y el papel de otros Estados en el proceso de migración forzada y reasentamiento.

Cuando se indagó sobre lo que sabían en relación con los actores del conflicto y motivos de la migración de su pueblo, los y las adolescentes identificaron a Marruecos como el principal causante del conflicto por la disputa del territorio, al parecer por el dominio y explotación de recursos naturales como el petróleo, fosfato y pesca del Sahara. Sumado a esto, expresaron que el gobierno marroquí ha usado técnicas de intimidación y violación de DDHH contra los saharauis en el momento de la ocupación del territorio como el envenenamiento del agua, 
siembra de minas antipersonal, encarcelamientos y asesinatos. Dos de las adolescentes entrevistadas dijeron conocer casos en su círculo familiar cercano de víctimas de este conflicto: una tía fue asesinada en la zona ocupada por el ejército marroquí y un hermano estuvo encarcelado también en la zona ocupada por portar la bandera saharaui.

Argelia es percibido por los y las participantes como el Estado que les ha proporcionado una solución "temporal" para poderse reasentar y Mauritania como otro actor en disputa por el territorio y los recursos naturales.

España aparece en el discurso de forma menos frecuente, sin embargo, hay un imaginario de España como un actor bueno o como Estado colonizador, frente a la dicotomía de ser percibido por algunos participantes como el Estado que vendió el Sahara a Marruecos "por tener muchas cosas bonitas" (Mohamed, ejercicio de escritura creativa, 4 de mayo de 2017). Asimismo, Francia aparece con una connotación negativa en las entrevistas por ser un actor interesado en el petróleo que ha apoyado a Marruecos.

Pese al claro reconocimiento de los entrevistados sobre el papel de Marruecos en todo el conflicto y cómo su accionar ha afectado la vida de su pueblo, ninguno de los participantes expresó sentimientos de resentimiento o rabia. Esto obedece, en parte, a que los adultos están transmitiendo continuamente a los adolescentes, la necesidad de buscar una solución pacífica al conflicto y mantener vivo el deseo de retorno.

Con la elaboración de la cartografía social se logró un acercamiento a su presente y la vida en los campamentos. En primera instancia, los y las participantes dieron a conocer los lugares que ellos consideraban más representativos de su wilaya. En este aspecto, se observó un acercamiento a la dimensión colectiva, determinada por prácticas culturales como celebraciones religiosas y culturales, así como por la vida en comunidad. 
Figura 1. Lugares representativos de Wilaya, según participantes

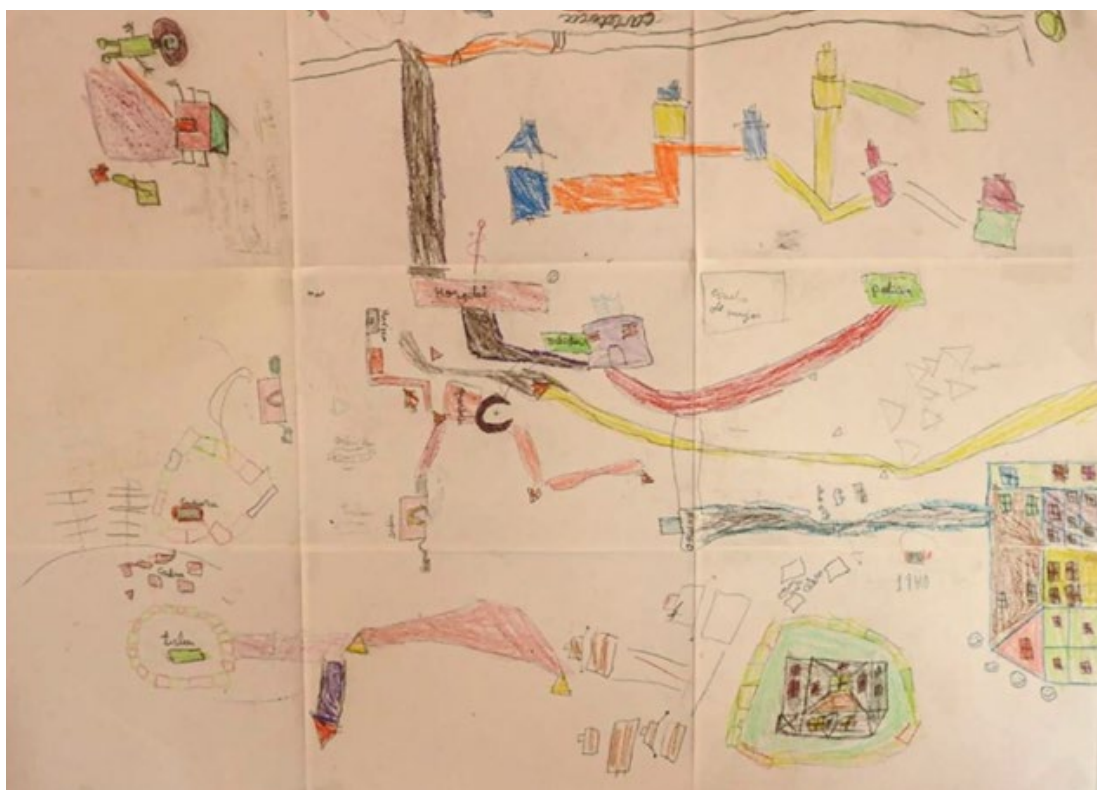

Fuente: elaborado por las participantes Tislem, Nayat, Nafi, Chej y Embarka

Cuando se indagó sobre las personas significativas en las dinámicas comunitarias cotidianas, el grupo resaltó la labor de los profesores y las mujeres, planteándose un reconocimiento al rol de las mujeres frente a las dinámicas organizativas del campamento. Sin embargo, cabe anotar que algunas de las participantes enfatizaron la importancia del papel de las mujeres en el ámbito familiar (doméstico), presentándose percepciones encontradas al respecto frente a las libertades de las mujeres, por sus creencias religiosas. En el guion del filminuto realizado por tres adolescentes mujeres, estas relatan:

La mujer saharaui es una mujer fuerte, independiente, segura de sí misma y de su capacidad, hace un buen aporte al campamento (...) Las mujeres tenemos un papel muy importante y es mantener el orden, no sólo en las haimas, sino en todo el campamento. Nuestra lucha ayuda a que sigamos en pie y soñemos con un futuro mejor (Embarka, Nayat y Tislem, taller de creación audiovisual, 10 de mayo de 2017). 
Aunque existen problemáticas, los y las participantes manifestaron sentirse seguros en los campamentos, percepción que va ligada directamente al hecho de que en el campamento no hay presencia marroquí que pueda hacerles daño.

Conviene aclarar entonces, la importancia que cobra la idea de retorno y su futuro en el discurso de los y las participantes. Expresiones como: "es mi país, cuando vivo ahí me siento más bien, porque veo mi tierra, siempre y cuando no haya presencia de marroquíes" (Bichu, grupo focal, 6 de mayo de 2017), evidencian su deseo de vivir en la zona ocupada. Empero, una participante que manifestó que: "me gustaría conocerlo porque es nuestro país de antes, conocerlo y así”, pero que no le gustaría vivir allá porque “ya estoy acostumbrada aquí, tanta pelea de allá no me gusta” (Nayat, grupo focal, 6 de mayo de 2017), de lo que se puede deducir las escasas posibilidades que ella vislumbra frente a la terminación del conflicto o el retorno a su tierra a corto o mediano plazo.

Para ellos, la lucha saharaui se centra en la recuperación de su territorio y el retorno de todo el pueblo lo cual está ligado a su idea de futuro, por eso, frente a la eventualidad del retorno, los participantes concuerdan en la importancia del reconocimiento de la soberanía de su país a nivel internacional y que puedan caminar por las calles de su pueblo portando su bandera libremente sin ser agredidos ni coartados.

\section{La construcción de la identidad de resistencia}

Si el territorio se configura como un espacio de encuentro donde confluyen y emergen comportamientos, así como prácticas individuales y colectivas, en el caso de los participantes, este concepto se puede asociar a la idea de territorio físico relacionado al campamento de refugiados, o simbólico cuando se refieren a los territorios ocupados.

Con referencia al espacio físico actual que habitan, se puede plantear este como un espacio liminal, en concordancia con Valdivia (2013). Allí han desarrollado estrategias de sobrevivencia, establecido una cotidianidad así como dinámicas sociales y comunitarias, fortalecidas por las prácticas culturales de su pueblo y su relación con el espacio habitado, lo cual se evidencia por ejemplo, en la importancia que tiene para los y las adolescentes la celebración y participación activa en las fiestas patrias como el 20 de mayo que es el día de la lucha por la causa del pueblo saharaui y el 27 de febrero donde conmemoran la proclamación de independencia de la República Árabe Saharaui Democrática.

En el grupo focal los y las participantes narraron al respecto de estas celebraciones: "El 20 de mayo todos los niños que decoren nos reunimos de todas las wilayas nos vamos a hacer esas fiestas del 20 de mayo, vamos a Awswert (wilaya) 
allá todos. Hay muchos extranjeros mucha gente” (Embarka, grupo focal, 6 de mayo de 2017).

Hay una (fiesta) que se hace el 27 de febrero, es la fiesta grande toda la gente disfrazada la parte de arriba de blanco y la parte de abajo como una falda de color negro nos ponemos una cosa que nos la ponemos en el pelo. Vamos disfrazados con una melfa negra arriba y desde aquí pones unas cosas como una falda, es una melfa pero hace de falda blanca y pones una cosa en el pelo, lo que se ponen en el pelo es sólo para las mujeres. (...) La fiesta del 27 de febrero va toda la gente a la plaza, cogen un hombre dicen cosas cantan (Tislem, grupo focal, 6 de mayo de 2017).

Acá, el sentido de permanencia transitoria en el campamento es visible y a su vez, el deseo y la idea de retorno a su país, pese a llevar toda su vida residiendo en este lugar y no tener certeza de cuánto tiempo más estarán allí.

En este contexto, el grupo en su totalidad denotó una actitud optimista frente al campamento como lugar de residencia, porque se sienten libres y seguros, y no menos importante porque allí están sus redes familiares y de amistad, lo cual, en este contexto es especialmente significativo, tanto individual (vínculos interpersonales y familiares) como de manera colectiva (tejido y lazos sociales en la comunidad), situaciones que se asocian a la resistencia con la que han sobrellevado el exilio en los campamentos.

Esto se evidencia en expresiones como "hemos luchado para eso, pero bueno hemos aguantado un montón, (...) siempre están juntos, para poder regresar” (Tislem, entrevista individual semiestructurada, 13 de mayo de 2017) y que "son buenas personas, para mí son las mejores personas" (Mariam, entrevista individual semiestructurada, 16 de mayo de 2017). Estas manifestaciones de solidaridad, unión, resistencia y empoderamiento que han sido fortalecidas en la adversidad son resultado de la capacidad de adaptación al entorno, los campamentos e interacción con el otro, en este caso, el otro refugiado, lo cual remite de nuevo al planteamiento de Castillejo (2000) con respecto a cómo se reconfigura el espacio del campamento a partir y en función de esos encuentros con los otros.

Frente al espacio simbólico, es decir la zona ocupada, se pudo evidenciar un vínculo alegórico cimentado en el arraigo y una forma de idealización basada primordialmente en lo que les han narrado (historias) y trasmitido (sentimientos) los adultos, evidenciado en la forma en que mantienen sus prácticas culturales (el baile, la música, la vestimenta, la comida, el té), la concepción de animales como las cabras y los dromedarios, como conciben la organización de su comunidad, las celebraciones religiosas y nacionales y el nombre de las wilayas haciendo referencia a las ciudades del RASD. Esto se evidencia en las respuestas 
que los y las participantes expresaron en el grupo focal cuando se planteó una comparación entre el campamento y la zona ocupada:

"Pues como la playa que allá si hay playa como casas, pisos (edificios de departamentos) que acá no ahî” (Nafi, grupo focal, 6 de mayo de 2017).

"Que es muy bonito que tiene casas bonitas y eso. Ahí árboles que acá en el campamento no hay. Aquí en el campamento hace calor, allá no hace calor, allá es otro país” (Elhga, grupo focal, 6 de mayo de 2017).

De esta manera, las adscripciones identitarias y el discurso colectivo con respecto a la historia del pueblo saharaui asociada a la descolonización española, el conflicto con Marruecos, la migración forzada y la llegada al campamento, eventos que los participantes no vivieron, en concordancia con Osorio (2004), son consecuencia directa del uso de prácticas culturales y la trasmisión de la memoria histórica de los adultos; ello, generó una interacción entre pasado y presente que ha permitido que quienes no estuvieron allí, tengan una versión de lo que ocurrió a pesar de la separación física, en este caso, de los territorios ocupados. Mariam, (grupo focal, 6 de mayo de 2017) expresa:

"Hay árboles acá no hay árboles, allá hay muchas cosas que aquí (campamento) no ahí. Allá hay playas y acá no hay, allá hay escuelas para los mayores que aquí no hay (...) Allá todo es bonito. Me han contado de la zona ocupada, me contó mi padre, que antes había casas y haimas, y tierras que tienen hierbas” (Mariam, 2017).

Si bien la memoria se construye de manera subjetiva, siempre está atravesada por narrativas colectivas en el marco de un contexto social. Así, podría plantearse que la memoria es el punto de encuentro del pueblo saharaui; esto nos permite hablar de la construcción de una memoria colectiva ligada a la idea del retorno, lo que orienta, además, su proyecto de vida.

En el caso del pueblo saharaui, se podría plantear que ante su historia de migración forzada y refugio, así como la respuesta de la comunidad internacional y ausencia de respuesta que den una solución duradera y satisfactoria a su exilio, han empezado a construir una identidad de resistencia, decidiendo actuar de manera colectiva ante su situación (inicialmente por la vida armada, actualmente por la vía política y empoderamiento pacifista) reconociéndose como sujetos protagonistas de sus propias historias con viabilidad de intervención voluntaria en todos los procesos sociales y políticos que atañen a su comunidad. 
Es importante recalcar, que los refugiados no pierden su identidad tras la migración forzada o el reasentamiento en un nuevo territorio, sino que, a partir de sus recuerdos y memoria histórica, junto con la reinserción en un espacio desconocido, generan nuevos núcleos de significado con relación a su presente y sentido del mundo, lo cual conlleva a la (re)configuración de sus procesos identitarios individuales y colectivos atravesados por procesos culturales, políticos y sociales en un contexto determinado por la pluralidad. Todo ello se convierte en una posibilidad de resignificar sus proyectos y sentidos de vida, así como de actualizar el pasado (lo que se era antes del acontecimiento) en las condiciones que posibilita el presente, con el fin de proyectarse en su futuro, redefiniendo su realidad dentro del campamento.

Las experiencias comunitarias de organización demuestran cómo los actores de la comunidad han recurrido a la identidad como defensa de su estructura social y política; el campamento se ha constituido como un espacio simbólico de resistencia donde se establecen numerosos puntos de encuentro, ya sea desde su situación de opresión o vulnerabilidad, o desde su empoderamiento y articulación de intereses comunes para superar estas situaciones creando un discurso propio en este contexto determinado, mientras pueden regresar al lugar que ellos consideran su territorio legítimo. Así, en consonancia con Alvarado, Patiño y Loaiza (2012) el territorio permite el surgimiento del sujeto político, dónde se actúa para transformarlo.

De acuerdo con la información recolectada, especialmente, en los filminutos y el ejercicio de fotoelicitación, para los y las adolescentes saharauis participantes, la transmisión de la memoria histórica se ha convertido en una herramienta para construir sus realidades y configurar sus identidades colectivas. Los saharauis frente a una situación conflictiva y de violencia directa, han desarrollado al máximo su capacidad de agencia, no sólo reorganizándose en un contexto desfavorable sino a nivel simbólico en el ámbito social y político. Todo esto es reconocido por los y las adolescentes participantes, en donde no se percibe ninguna intención de revictimizarse, sino por el contrario, se reconocen como sujetos agentes de cambio con capacidades para aportar al retorno, así como en el reconocimiento de su país como un Estado soberano.

Lo anterior, se soporta con la postura de Jelin (2002) quien señala que el pasado no invade el presente, sino que lo informa, fortaleciendo el sentido de pertenencia del individuo y las comunidades, lo cual se incrementa en grupos oprimidos, vulnerados y discriminados. De esta manera, los y las adolescentes tienen clara y presente su condición de refugiados ligada a la idea de territorio y el retorno, tal y como lo explica una de participantes "porque vivo en un pueblo que no es mío" (Embarka, grupo focal, 6 de mayo de 2017). 
Lo más interesante que se detectó en los discursos expuestos por los y las adolescentes, es lo que consideran correcto para materializar el retorno de su pueblo. En su totalidad coincidieron que la mejor forma es la vía pacífica, deslegitimizando las acciones violentas que se hayan podido presentar en el conflicto. $\mathrm{Al}$ respecto, una participante expresó no querer hacer parte de la lucha saharaui (la cual para ella tiene una connotación militar), pero manifiesta como solucionaría el conflicto:

Solucionaría el conflicto, que todos los pueblos que van con Sahara para ganar la lucha, invitaría a España, a Argelia y muchos (...) Les diría podéis ayudarme que mi pueblo está ocupado y quiero ganar la guerra. Pero yo no quiero matar nadie, sólo quiero decir que, si pueden salir de mi pueblo, que yo quiero vivir feliz" (Tislem, entrevista individual semiestructurada, 13 de mayo de 2017).

En el grupo focal, dos participantes establecieron una relación entre la lucha saharaui y su participación en ella por medio de las ideas y su formación académica: "Me siento parte de la lucha saharaui y aportaría a ella con ideas" (Mohamed, grupo focal, 6 de mayo de 2017), "tengo que estudiar para ayudar a mi pueblo y así estoy ayudando a ello”, (Mariam, grupo focal, 6 de mayo de 2017). Finalmente, una participante expresó: "es mi país, cuando vivo ahí me siento más bien, porque veo mi tierra, siempre y cuando no haya presencia de marroquíes" (Bichu, grupo focal, 6 de mayo de 2017), donde se percibe el deseo genuino de regresar a la zona ocupada siempre y cuando sus compatriotas no tengan enfrentamientos violentos con los marroquíes de nuevo.

Pese al reconocimiento de los actores responsables de su situación de refugio, no se perciben sentimientos de rabia, venganza u odio hacia estos en sus discursos, al contrario, se percibe lo que Jelin (2002) denomina un propósito político y educativo por medio de la transmisión de la lucha política, que da cuenta de los actos represivos de los cuales ha sido víctima su pueblo, mostrando otros caminos alternativos que posibiliten la no repetición de actos violentos.

En el caso del pueblo saharaui, se podría plantear que ante su historia de migración forzada y refugio, así como la respuesta de la comunidad internacional y ausencia de respuesta que ofrezcan una solución duradera y satisfactoria a su exilio, este grupo de adolescentes ha empezado a configurar una identidad de resistencia, reconociéndose como sujetos protagonistas de sus propias historias con viabilidad de intervención voluntaria en procesos sociales y políticos que atañen a su comunidad, especialmente en lo relacionado con el retorno a su territorio. De esta manera se establecen numerosos puntos de encuentro, ya sea desde su situación de opresión o vulnerabilidad, o desde su empoderamiento 
y articulación de intereses comunes para superar estas situaciones creando un discurso propio en este contexto.

Estas acciones, evidenciadas en sus prácticas discursivas en relación con el contexto e historia del pueblo saharaui, se pueden reconocer desde el paradigma de paz imperfecta y enmarcar como procesos de empoderamiento pacifista, alimentados por la configuración de las identidades colectivas, en este caso de resistencia. El pueblo saharaui por medio de su organización comunitaria, social y política ejerce el poder de manera noviolenta para transformar su realidad de manera positiva, decidiendo actuar de manera colectiva ante su situación (inicialmente por la vida armada, actualmente por la vía política y empoderamiento pacifista), garantizar sus derechos y evitar su continua vulneración, así como, para tener mayor incidencia política y pública, no sólo visibilizando su situación sino trasmitiendo este discurso a las nuevas generaciones, tal como lo plantea Muñoz (2001), con intención e interés en promocionar la paz.

\section{Conclusiones}

Comprender el refugio desde una perspectiva de paz implica, como paso primordial, reconocer el empoderamiento de los sujetos protagonistas, el cual se ve fortalecido por diferentes procesos identitarios tanto a nivel personal potenciando su propia subjetividad (proceso interior de auto dignificación y reconocimiento), como grupo social que legitime y reivindique de manera colectiva y protagónica su historia y lugar en la sociedad. Se podría decir que la experiencia de este campamento es un ejemplo de materialización de la categoría analítica de la paz imperfecta en la práctica, como un espacio y experiencia donde los diferentes actores de la comunidad han decidido regular y transformar pacíficamente el conflicto, haciendo hincapié en la necesidad de reconocer las aportaciones no violentas de cada uno.

En este proceso de hacer las paces enmarcado en la lucha saharaui, la trasmisión de la memoria histórica de los adultos a las generaciones más jóvenes, se ha constituido en el eje central para la configuración de una identidad colectiva de resistencia y la consolidación de procesos de empoderamiento pacifista en este grupo de adolescentes. Esto ha permitido no sólo el conocimiento de la historia del conflicto del pueblo saharaui y de los actos represivos y de vulneración a sus derechos, sino que ha construido una conexión entre presente y pasado de no repetición de estos actos de violencia, basado en un sistema de valores y principios relacionados con su identidad, sumado al desarrollo prácticas discursivas claras y consistentes de resistencia y noviolencia frente a las formas de dominación con relación a su futuro, el cual está directamente ligado al retorno. 
La construcción de la realidad de los y las adolescentes en su entorno inmediato, en diálogo con el espacio y las relaciones y dinámicas sociales, está mediada por su cultura, creencias y transmisión de la memoria, lo cual ha fortalecido el vínculo simbólico con su territorio de origen (zona ocupada) y ha permitido resignificar el territorio de asentamiento actual (campamento). De esta manera se abre el espectro para reconocer la singularidad de los procesos de cada comunidad refugiada, en este caso la saharaui, con un enfoque identitario, así como la pluralidad de las narrativas y procesos sociales de empoderamiento, lo cual permitirá romper con la homogenización de la experiencia del refugio unida a la clasificación de víctimas.

Por esto, se reitera la importancia de reconocer el proceso de empoderamiento pacifista de la comunidad saharaui como una acción propia del paradigma de la paz imperfecta, así como la visibilización de estas experiencias de paz de una comunidad históricamente excluida y violentada, para aportar al reconocimiento social de las mismas, generando esperanza, cambio cultural y movilizando al otro(s) a participar de estas prácticas. Así, la historia de resistencia del pueblo saharaui se constituye como un ejemplo de construcción de paz mundial, pueblo que se reconoce y construye cotidianamente desde perspectivas locales y prácticas comunitarias y culturales pacíficas, así como en la trasmisión y fortalecimiento de su tejido social basado en valores como la cooperación y la solidaridad. Por consiguiente, el pueblo saharaui ha demostrado la posibilidad y capacidad que tienen todas las personas y grupos sociales de ser actores continuos, momentáneos o coyunturales de la construcción de paces.

En el caso de los y las adolescentes participantes, se logró identificar como gracias a la transmisión de la memoria histórica han asumido su realidad desde valores culturales y potenciación de sus capacidades, construyendo prácticas discursivas fortalecidas en una decisión política basada en respuestas noviolentas, y en un enfoque de poder pacifista, configurándose en una experiencia en el marco del paradigma de la paz imperfecta, donde la paz aun inacabada se construye día a día, desde abajo y horizontalmente. 


\section{Referencias}

Abdala, S. (2017). Prácticas educativas inclusivas en la República Árabe Saharaui Democrática (RASD) (Tesis Doctoral). Universidad Central "Marta Abreu" de Las Villas, Cuba.

Anacona, A. (2019). Memoria del proceso de empoderamiento pacifista de las mujeres del cabildo indígena Yanakuna Santiago de Cali 1999 a 2014 (Tesis doctoral, inédita). Universidad de Granada, España.

Alvarado, S.; Patiño, J.; Loaiza, J. (2012). Sujetos y subjetividades políticas: El caso del movimiento juvenil Álvaro Ulcué. Revista Latinoamericana de Ciencias Sociales, Niñez y Juventud, 1O(2), 855-869. https://www.redalyc.org/pdf/773/77323978006.pdf

Aranda, R.; Arias, S.; González, L. (2012). La formación del profesorado saharaui en los campamentos de refugiados de Tindouf (Argelia). Tendencias Pedagógicas, 20, 140-159. https:/ / revistas.uam.es/tendenciaspedagogicas/ article/view/2019

Bartolomé, M. (1990). La investigación cooperativa. En M. Bartolomé y M. Anguera. La investigación cooperativa una vía para la innovaciónen la Universidad. PPU.

Berger, P.; Luckmann , T. (1995). Modernidad, plurarismo y crisis de sentido. La orientación del hombre moderno. Ediciones Paidós Ibérica.

Berger, P.; Luckmann, T. (1999). La construcción social de la realidad. Talleres Gráficos Color Efe.

Boulding, K. (1993). Las tres caras del poder. Editorial Paidós.

Calero, M., Cruz, A.; Campos, A. (2016). La mejora de la salud de la población saharaui a través de la innovación docente en educación: Un proyecto de formación en cascada. Index de enfermería, 25(3), 198-202. https:// www.semanticscholar.org/paper/La-mejora-de-la-salud-de-lapoblaci\%C3\%B3n-saharaui-a-de-Garc\%C3\%ADa-Lend\%C3\%ADnez/ bd46dc8fc9cf54561565e6012678de22e96ced 87 
Canales, D. (2009). Imaginación y reacción en los Campamentos de refugiados saharauis. construcción de la cotidianeidad e identidad frente al otro extranjero (nasrani) en el contexto de la diáspora. Perifêria: Revista de investigación y formación en antropología, 10, 1-24. http://dx.doi.org/10.5565/rev/periferia.523

Carrizosa, C. (2011). El trabajo de la memoria como vehículo de empoderamiento político: La experiencia del Salón del Nunca Más. Boletín de Antropología, 25, 33-56. https://revistas.udea.edu.co/index.php/boletin/article/ view/11224/10290

Castells, M. (1999). La era de la información. Economía, sociedad y cultura. El poder de la identidad. Siglo Veintiuno Editores.

Castillejo, A. (2000). Poética de lo otro. Antropología de la guerra, la soledad y el exilio interno en Colombia. Instituto Colombiano de Antropología e HistoriaICANH, Ministerio de Cultura y Colciencias.

Colangelo, M. (2014). La crianza como proceso sociocultural. Posibles aportes de la antropología al abordaje médico de la niñez. Primeras Jornadas Diversidad en la Niñez, 1-12. https://www.aacademica.org/o00-098/6.pdf

Egea, C.; Soledad, J. (2011). Los desplazados ambientales, más allá del cambio climático. Un debate abierto. Cuadernos Geográficos, 49, 201-215. https://www.redalyc.org/pdf/171/17122051008.pdf

Fisas, V. (1998). Una Cultura de Paz. En V. Fisas, Cultura de paz y gestión de conflictos (pp. 349-350). Icaria/NESCO.

Gimeneo, J.; Ali, M. (2007). Transformaciones socioculturales en los campamentos de refugiados Saharauis al sur de Argelia, 1975-2005. En J. Gimeneo, O. Mancha, A. Toledo (Eds.). Conocimiento, Desarrollo y Transformaciones Sociales. Sepha.

Giménez, G. (2000). Identidades étnicas: estado de la cuestión. En L. Reina. Los retos de la etnicidad en los Estados-nación del siglo XXI (pp. 61-64). Instituto Nacional Indigenista, M.A. Porrúa Grupo Editorial. 
Giménez, G. (2009). Cultura, identidad y memoria. Materiales para una sociología de los procesos culturales en las franjas fronterizas. Frontera Norte, 21(41), 7-32. http://www.scielo.org.mx/pdf/fn/v21n41/v21n41a1.pdf

Girela, B. (2016). Procesos de mediación y empoderamiento pacifista en el barrio Candeal de Salvador de Bahía (Brasil). Revista de Paz y Conflictos, 9(1), 115131. Granada, España: Universidad de Granada.

Hernández, E. (2014). Empoderamiento pacifista de experiencias comunitarias locales en Colombia (1971-2013) (Tesis doctoral). Universidad de Granada. España. https://hera.ugr.es/tesisugr/24287106.pdf

Hernández, E. (2015). Empoderamiento Pacifista del actual proceso de paz en Colombia: 2012-2015. Revista de Paz y Conflictos, vol. 8, núm. 2, 2015, pp. 179-202. Universidad de Granada. España. http://www.redalyc.org/ articulo.oa?id=205043417008

Hodges, T. (1984). The western Saharans. Minority Rights Group. http://www.pensamientocritico.org/tonhod 1114.pdf

Jelin, E. (2002). Los trabajos de la memoria. Siglo Veintiuno de España editores.

Jiménez, A. (2015). El español en los campamentos de refugiados Saharauis de Tinduf: práctica docente (Tesis Doctoral, inédita). Universidad de Granada, España.

Loaiza, J. (2016). Niños, Niñas y Jóvenes Constructores-as de Paz. Una experiencia de Paz Imperfecta desde la potenciación de subjetividades politicas (Tesis Doctoral, inédita). Universidad de Manizales - CINDE, Manizales, Colombia.

López, M. (2011). Reflexiones sobre las desigualdades en el contexto de los estudios de paz. Revista Paz y Conflictos, 4, 1-15. https://www.ugr.es/ revpaz/tesinas/rpc_n4_2011_dea2.pdf

Mato, D. (2000). Not "Studying the Subaltern", but Studing with "subaltern" social Groups, or, at least, Studying the Hegemonic Articulation of Power. Nepantla: Views from teh South, 3(3), 479-502. http://www.scielo.org.co/ scielo.php?script=sci_arttext\&pid=S0124-40352014000100008

Mayor, F. (1994). La nueva página. UNESCO/Círculo de Lectores. 
Muñoz, F. (2001). La paz imperfecta. Editorial Universidad de Granada.

Muñoz, F; Martínez, C. (2011). Los habitus de la paz imperfecta. En F. Muñoz, \& M. Bolaños. Los habitus de la Paz: Teorías y prácticas de la paz imperfecta (pp. 37-64). Granada, España: Universidad de Granada. https://dialnet.unirioja.es/servlet/libro?codigo $=476911$

Osorio, F. (2004). Recomenzar vidas, redefinir identidades: algunas reflexiones en torno a la recomposición identitaria en medio de la guerra y del desplazamiento forzado. En M. Bello, Desplazamiento forzado. Dinámicas de guerra, exclusión y desarraigo (pp. 175-186). Acnur - Universidad Nacional de Colombia.

Parra, L. (2014). Prácticas y experiencias colectivas ante la guerra y para la construcción de paz: Iniciativa sociales de paz en Colombia. El Ágora USB, 14(2), 311- 703. http://www.scielo.org.co/pdf/agor/v14n2/v14n2a02. pdf

Portillo, L. (2014). Los Saharauis (The Western Saharans). http://www.pensamientocritico.org/tonhod 1114.pdf

Posada, J.; Briceño, P.; Munar, Y.; Corredor, N.; Rossi, J. (2018). Subetividades políticas de paz en jóvenes de Colombia. Revista Aletheia, 1O(1), 148-173. http://dx.doi.org/10.11600/21450366.10.1aletheia.148.173

Pozuelo, L.; Gimeno, J. (2010). Expectativas y desafíos para la transformación social de refugiados saharauis: Expectativas y desafíos para la transformación social. VII Congreso Ibérico de Estudios Africanos, (pp. 1-21). https://repositorio.iscte-iul.pt/bitstream/10071/2284/1/CIEA7_8_ MART\%C3\%8DN\%26GIMENO_Renovaci\%C3\%B3n\%20del\%20 Plan\%20de\%2oFormaci\%C3\%B3n\%20Profesional.pdf

Refworld (1984). Declaración de Cartagena sobre los Refugiados. Adoptada por el "Coloquio Sobre la Protección Internacional de los Refugiados en América Central, México y Panamá : Problemas Jurídicos y Humanitarios. https://www.refworld.org.es/docid/50ac93722.html 
Romariz, Y. (2012). Renunciar a la herencia del pasado. Revista Migraciones Forzadas (RMF), 40, 1-6. https://www.fmreview.org/sites/fmr/files/ FMRdownloads/es/pdf/RMF40/RMF 40\%20Jovenes\%20y\%20fuera\%20 de\%2olugar.pdf

Salas, W.; Valenzuela, E.; Prada , A. (2019). Experiencias significativas en procesos de construcción de paz en tres municipios del Magdalena Medio afectados por la violencia. El Ágora USB, 19(2), 323-342. http://dx.doi.org/10.21500/16578031.3944

Sandoval, E. (2009). Ciudadanía universal, derechos políticos y paz en la migración. En V. Martínez y E. Sandoval, Migraciones, conflictos y cultura de paz (pp. 19-44). Bonobos Editores.

Sandoval, E. (2015). Empoderamiento pacifista para otros mundos posibles. Revista de Paz y Conflictos, 8(2), 75-95. https://revistaseug.ugr.es/index. $\mathrm{php} / \mathrm{revpaz} / \mathrm{article} / \mathrm{view} / 3312$

Santander, P. (2011). Por qué y cómo hacer Análisis de Discurso. Cinta moebio 41, 207-224. www.moebio.uchile.cl/41/santander.html

Valdivia, O. (2013). Espacio e identidad en campamentos de refugiados: Experiencia del grupo musical Sierra Leone's Refugee All Stars. Migraciones Internacionales, 7(2), 127-155. http://www.scielo.org.mx/ scielo.php? script $=$ sci_arttext\&pid $=\mathrm{S} 1665-89062013000200005$ 
